\title{
ÍNDICE CIENTÍFICO DA CIÊNCIA DA INFORMAÇÃO: COMPARATIVO DOS AUTORES COM MAIOR VISIBILIDADE NO ISI E NO GOOGLE ACADÊMICO
}

\section{CONTENIDO DE LA CIENCIA DE LA INFORMACIÓN: COMPARATIVO DE LOS AUTORES MÁS VISIBLES EN EL ISI Y EN EL GOOGLE ACADÉMICO}

\begin{abstract}
Adilson Luiz Pinto - adilson.luiz2208@hotmail.com
Doutor em Documentación pela Universidad Carlos III de Madrid. Consultor de Análise de Redes e Estudos Bibliométricos, Calle - Madrid

João de Pontes Junior - jpontesjunior@yahoo.com.br Mestrando em Ciência da Informação pela Pontifícia Universidade católica de Campinas (PUC-
\end{abstract} Campinas).

\section{Resumo}

Este trabalho analisou a produtividade dos principais autores da área de Ciência da Informação no ISI e Google Acadêmico, utilizando uma metodologia de recuperação da informação por país, seguida de um filtro por subcategorias (ISI) e por autoria (Google Acadêmico). Para o refinamento dos dados utilizamos ProCite para o ISI e para o ambiente web o FlashGet. Os resultados ressaltam a alta produtividade dos autores da UFRJ e da Unicamp, assim como destacam o elevado índice de citação dos autores da UFRJ. A colaboração científica entre os autores está representada em forma de mapas de acordo com as redes sociais a que pertencem.

\section{Palavras-chave}

Visibilidade científica; ISI; Google Acadêmico; Análise de citação; Rede Social. 


\section{INTRODUÇÃO}

Os seres humanos mostram uma constante preocupação por visualizar a natureza da informação, procurando descrever sua evolução em detalhes e suas necessidades, através das características fundamentais no que tange a sua busca. O segredo de uma boa busca informacional está em sua ordem e sua lógica booleana, principalmente quando estas informações estão em ambiente web (MARCHIONINI, 1995).

Com a expansão e a grande quantidade de informação disponível na Internet se fez primordial a aparição de diretórios e motores de busca. Os diretórios possuem a função de organizar os links com outros sites na web, sendo necessário que seus desenvolvedores informem descritores durante a confecção para que ele seja incluído, e conseqüentemente os editores autorizados a revisarem essas solicitações antes de incluir seus links para comprovar sua adequação (GOTO e COTLER, 2005), um exemplo é o Yahoo. A função dos motores de busca é trabalhar com palavras-chave (linguagem natural), como uma base de dados, incorporando automaticamente páginas da web mediante robôs de busca na rede (LELOUP, 1998), como por exemplo, o Google.

Em relação aos motores de busca, estamos passando pela quarta geração, com maior conteúdo e com aplicativos especializados ao universo acadêmico, como a criação do Google Acadêmico em 2004, (GOOGLE, 2005), que representa um buscador especializado no mundo acadêmico, focado por um lado em publicações de documentos realizados na web, citações recebidas e tipologias documentais (JACSO, 2005). Sua função é hierarquizar os resultados usando um algoritmo similar ao que utiliza o Google para as buscas gerais, utilizando também ferramentas especificas que refinam a pesquisa deixando-as com qualidade, permitindo encontrar as informações solicitadas com maior precisão e atualização (GUTIÉRREZ GALLARDO e LÓPEZ GUISADO, 2005), pode ser também um excelente recurso estatístico para averiguação e comparação científica de um país, instituição e autoridade (NORUZI, 2005).

O motor de busca Google Acadêmico tem uma função extra que é disponibilizar ao usuário o número de citações que cada documento recebe durante sua existência na Internet, onde aparecem indicadas todas as tipologias bibliográficas.

A análise de citações é um dos critérios que se emprega com maior freqüência para a seleção da literatura científica (PRICE, 2007). As análises de citação são muito utilizadas na seleção de informação científica para o 
desenvolvimento de coleções, estudos de fundo, assim como para a colaboração científica e para o consumo de informação dentro de uma comunidade científica. Constitui-se como uma das ferramentas mais utilizadas tanto para a avaliação das publicações como da atividade científica (CAÑEDO ANDALIA, 1999a). Seu uso neste contexto serve de avaliador das fontes bibliográficas que foram utilizadas.

Através da análise de citações podese conhecer a intensidade do emprego de uma revista ou da obra de um referido autor o que proporciona uma medida razoável de sua importância científica.

A utilização das citações bibliográficas - como critério de avaliação dos artigos e das publicações científicas fundamenta-se em seu caráter condicionante das principais etapas da investigação científica. Coloca-se aqui a importância da realização de uma revisão bibliográfica exaustiva, imparcial, representativa e atualizada, sob critérios objetivos de valorização e seleção tanto para o processo de investigação como para a avaliação dos trabalhos científicos (CAÑEDO ANDALIA, 1999b).

Por outro lado à citação no Google Acadêmico pode ser um fator importante num comparativo para países de menor inferência científica nas grandes bases de dados, como no caso do ISI (BAR-ILAN, LEVENE e LIN, 2007). Este processo de contrastar o potencial de um país, ou até mesmo de uma autoridade é intrigante, mesmo porque até alguns anos não imaginávamos que a Internet, com suas duplicações de documentos e uma indexação supostamente descontrolada, poderia ser ou ter um indicador científico, isso até a chegada os motores de quarta geração (Google Acadêmico e Scirus).

Partindo destas informações objetivamos desenvolver um estudo sobre a produção e a citação de alguns autores da área de Ciência da Informação brasileira dentro do Google Acadêmico e dos produtos do ISI, onde selecionamos os 10 autores mais representativos nas bases de dados do Instituto for Scientific Information e identificamos seu índice científico e de citação, realizando posteriormente o mesmo processo no domínio acadêmico do Google, como uma comparativa, verificando se este recurso web pode ser aplicado para uma análise científica nacional.

\section{METODOLOGIA}

Este estudo analisou a produtividade e o índice de citação dos 10 primeiros autores nos produtos do Institute for Scientific Informaction (Science Citation Index, Social Science Citation Index e Arts \& Humanities Citation Index) na subcategoria Information Science \& Library Science, e posteriormente utilizou-se estes 
mesmos autores para poder comparar seu índice frente ao Google Acadêmico, identificando seu ranking científico e sua produtividade.

Para esta pesquisa não utilizamos um período de estudo, porém dentro do ISI os documentos mais antigos foram de 1994 e para Google Acadêmico foi de 1987. Para a recuperação da informação no ISI utilizamos uma busca por país (CU=Brazil), depois refinados para a sub-categoria Information Science \& Library Science, e posterior um refinamento pela subcategoria Authors, onde conseguimos o índice dos autores com maior produção científica.

O passo seguinte foi identificar os 10 mais importantes e realizar uma busca por autores, onde também selecionamos a sub-categoria Information Science \& Library Science, no qual conseguimos identificar diferenças quantitativas nos dois tipos de busca.

Para Google Acadêmico utilizamos uma estratégia inicial com o nome dos 10 autores mais importantes em ISI, refinando a busca por nome completo dentro de aspas e depois comparados por uma busca especial dentro desta ferramente web (autor:j-leta), que conseguimos uma fusão mais adequada e com um resultado melhorado e mais confiável.

Em ambos os recursos utilizamos um filtro posterior para poder identificar o solapamento de registros, no qual podemos afirmar que dentro do ISI não foi identificado nenhuma duplicação e, no Google Acadêmico conseguimos visualizar 3 duplicações em todo o processo, isso porquê esta última ferramenta (web) contém um controle para solucionar este problema em sua estrutura.

Dentro da recuperação de dados conseguimos obter um total de 82 registros em ISI, sendo todos artigos de revista, e para Google Acadêmico recuperamos 545 registros entre artigos científicos e trabalhos apresentados em congressos, 9 livros e 259 documentos mencionados por [CITAS], com um total de 813 registros.

Para o tratamento dos registros recuperados em ISI utilizamos o software ProCite (SAXTON, 2001) para a análise dos autores e dos documentos, fator relevante na escolha do software pelo fato de que o ISI-CE aceita muito bem o programa e é adequado para o processo de documentos indexados em suas bases. Para a recuperação e tratamento das informações em Google Acadêmico utilizamos FlashGet versão 1.9.2.1028 com suporte do Internet Explorer, conseguindo exportar todos os dados para um arquivo em formato XLS.

Em relação à representação gráfica das colaborações científica, utilizamos o CreatePajek, neste caso os dados também foram transferidos para um arquivo no 
formato XLS para uma linguagem em brasileiros fazem parte (MOLINA, MUÑOZ .NET, em seguida utilizamos o Pajek e DOMENECH, 2001); e uma análise das (BATAGELJ e MRVAR, 2003) para a citações recebidas pelos autores no âmbito conversão em imagem. pessoal por autoria e por documentação (GARFIELD, 1955).

\section{RESULTADOS}

Para a produtividade científica dos

O contexto dos resultados foi planificado em três partes, iniciando uma averiguação da produtividade científica e o grau de colaboração em ambos os recursos bibliográficos; um comparativo do universo das Redes em que os 10 autores autores podemos dizer que somente um dos autores (Woodall, J.) teve praticamente o mesmo índice em ambos os recursos, os demais tiveram um percentual muito mais elevado no Google Acadêmico, conforme visualizado no Quadro 1.

\begin{tabular}{|c|c|c|c|c|c|}
\hline \multirow{2}{*}{ Autor } & \multirow{2}{*}{ Instituição } & \multicolumn{2}{|r|}{ ISI } & \multicolumn{2}{|c|}{ Google Acadêmico } \\
\hline & & Qt & Colaboração & Qt & Colaboração \\
\hline Woodall, J & UFRJ & 17 & & 19 & 11 \\
\hline Leta, J & UFRJ & 11 & 10 & 43 & 43 \\
\hline Sabbatini, R.M.E & UNICAMP & 10 & 10 & 110 & 42 \\
\hline Novaes, M.A. & UFPE & 7 & 6 & 48 & 47 \\
\hline De Meis, L. & UFRJ & 6 & 6 & 13 & 10 \\
\hline Rocha, B.H.S.C. & UFPr & 7 & 7 & 251 & 218 \\
\hline Cardoso, S.H. & UNICAMP & 5 & 5 & 71 & 47 \\
\hline Marin, H.F. & UNIFESP & 5 & 5 & 115 & 88 \\
\hline Ribeiro-Neto, B.A. & UFMG & 5 & 5 & 42 & 41 \\
\hline Rocha, R.A. & Intermountain HIth Care & 9 & 9 & 101 & 97 \\
\hline
\end{tabular}

Quadro 1- Produtividade e colaboração em ISI e Google Acadêmico. 
A proporçao de ambos os recursos tol muito distinto, justificado pelo fato de que nas bases de dados do ISI os materiais divulgados passam pelo filtro das revistas indexadas, que são na grande maioria as "melhores revistas" das áreas, e no caso da Ciência da Informação não poderiam ser diferente. Dentro da análise podemos destacar: Scientometrics, Scientist, Social Science Information Sur Les Sciences Sociales, Journal of the American Medical Informatics Association, Telecommunications Police, Journal of Information Science, International Journal of Information Management, Journal of the American Society for Information Science and Tecnology, Knowledge Organization, Information Research-An International Electronic Journal, International Journal Geographical Information Science, Journal of Librarianship and Information Science, Information Society, Libri entre outras.

Em relação às revistas ISI identificamos a falta de publicações que consideramos importantes para a área em nível internacional, como Journal of Documentation e Annual Review Information Science and Technology, porém podemos afirmar que a visibilidade de revistas da área de Ciência da Informação está bem representada.

$\mathrm{Na}$ parte da análise no Google Acadêmico a visibillidade foi maior pelo fato de que todas as revistas estão
Indexadas na web parcialmente (com o sumário e os artigos) ou completamente, sendo o grande elo entre as publicações e seu público. Para a visibilidade em números destacamos as seguintes publicações: ACM Sigmod Records, Journal Biological Chemical, Journal of the American Medical Informatics Association, Annual Review Biomedical, Behavioral and Brain Review, Biochemistry, Biochimica et Biophisyca Actas, Computers and Biomedical Research, Example-Based Environment for Wrapper Generation, Neuroscience and Biobehavioral Reviews, Physiology \& Behavior, Processing Third ACM Conference Digital Library, Review Informédica, Scientometrics e Brazilian Journal of Medical and Biological Research.

Nesta averiguação podemos afirmar que as literaturas da área de Biblioteconomia, Arquivo e Documentação são muito baixas, onde identificamos uma forte influência das áreas de Biologia, Química, Medicina e área tecnológicas em função das profissões e das investigações dos autores mais produtivos.

No que confere a colaboração científica das publicações em ambas as fontes de informação averiguamos que os autores Leta, J.; Noaves M.A.; De Meis L.; Rocha, B.H.S.C.; Ribeiro Neto, B.A. e Rocha, R.A. tiveram um índice muito equiparados em ambos recursos, porém 
Woodall, J.; Sabbatini, R.M.E.; Cardoso, identificamos nove "clusters" com

S.H. e Marin, H.F., tiveram uma proporção maior para somente um recurso, no outro a colaboração foi nula ou abaixo da outra fonte informacional.

Um dado curioso dos autores representados é que alguns deles trabalham diretamente com outros grupos de pesquisa ou fazem parte do mesmo departamento de investigação, como os autores representantes da UFRJ e da Unicamp.

Para uma melhor aproximação desta informação foi estudada a Rede Social dos autores para poder identificar quais são os seus parceiros científicos sobre dois pontos cruciais, (i) de colaboração pessoal e, (ii) de interligação entre dois ou mais "clusters".

Em relação à representação, desenvolvemos dois mapas de relações com a mesma estrutura e posição geográfica dos autores, onde foi visualizado primeiramente em ISI (Figura 1) e posteriormente em Google Acadêmico (Figura 2).

Para a averiguação nos produtos do Institute for Scientific Information centralidade de somente um autor (todos os autores da Quadro 1 com excessão de Woodall) e três "clusters" com vários autores que intermediaram os "clusters" maiores (representados por setas). Somente um "cluster" ficou isolado dos demais (Ribeiro Neto BA), sem nenhum elo com outro, porém existiu um "cluster" centrado por Cardoso, S.H. que todas as suas colaborações estiveram também vinculadas ao grupo de Sabbatini, R.M.E.

$$
\text { Os "clusters" intermediários, }
$$
representados pelo círculo maior, são sem sombra de dúvida os dois mais importantes desta análise, porque ambos "clusters" concentram um número elevado de autores e que, na realidade, estes autores fazem parte de dois ou três clusters identificando que diretamente estes autores, no caso Rocha BHSC, Rocha RA e Marín HF, estão desenvolvendo projetos em colaboração entre eles ou através de outros autores que estão intimamente ligados. O mesmo passou pelos autores Jacques $R$, Lannes $D$ e Figueira I que unem os clusters de Jacqueline Leta e Leopoldo de Meis. 


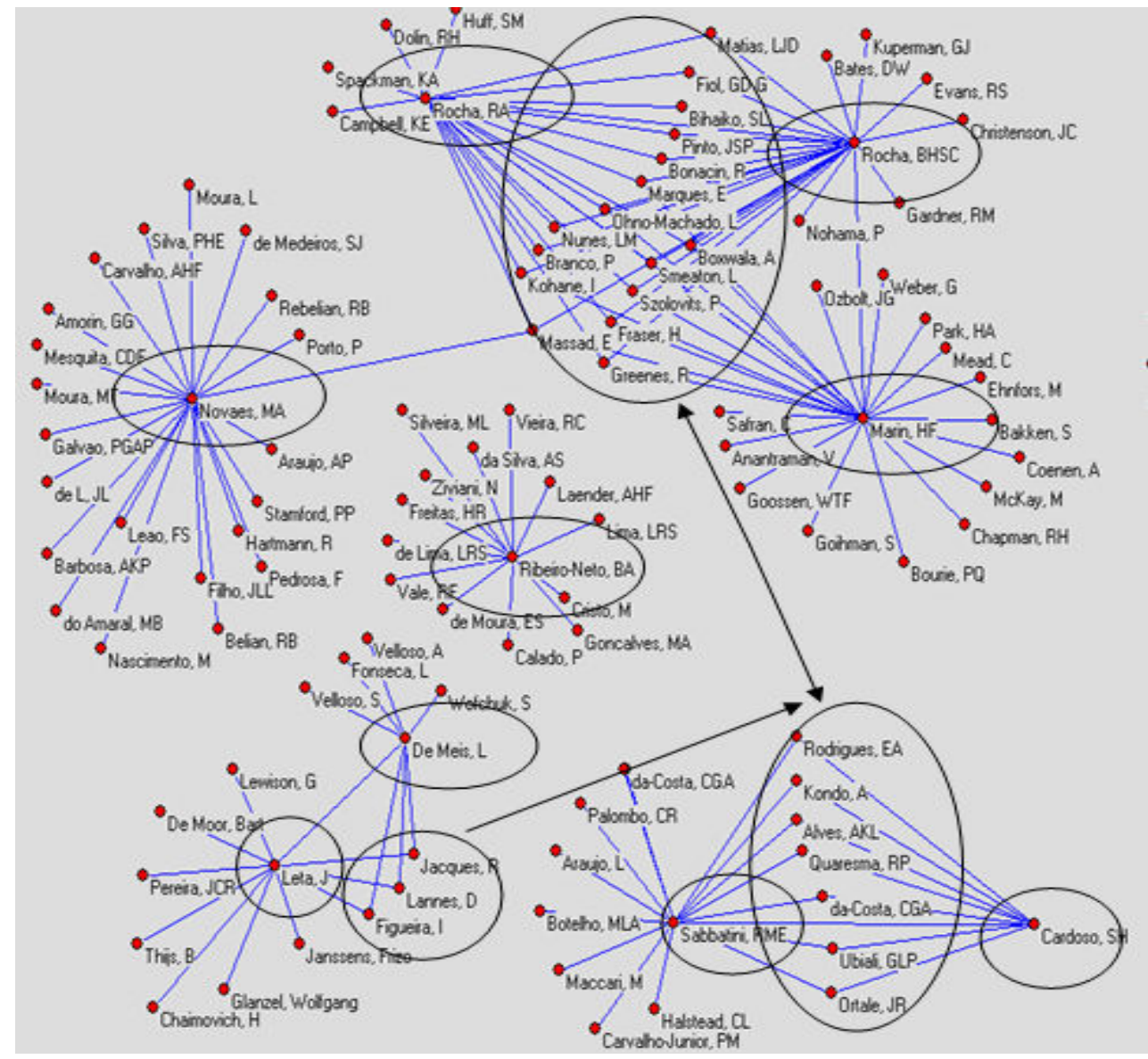

Figura 1 - Rede Social dos autores em ISI.

Para a análise no Google Acadêmico definimos que dentro dos trabalhos mais importantes dos autores (que figuram na Quadro 1) criamos uma rede de colaboração, onde representamos dez grupos de autores centro e mais um grupo de autores intermediário.

É importante observarmos um detalhe para esta análise, que não utilizamos todos os trabalhos mencionados na lista dentro do Google Acadêmico, somente utilizamos os 100 mais importantes (em número de citações recebidas) com mais de uma autoria.
Neste caso não temos a constante relação intermediada por autores entre Sabbatini, Cardoso, Marin, Leta e De Meis, onde esta ligação é representada pelos mesmos autores. A única intermediação latente é representada entre Rocha, B.H.S.C. e Rocha, R.A. através de Haug, Fiol e Huff.

O número de relações dos autores chave (de centralidade) foi bem intenso, como a análise anterior (Figura 1), porém é importante mencionar que mesmo o autor Woodall tendo uma pequena colaboração mostra que dentro das especificações da área estudada em ISI este autor não 
buscou relações científicas, entretanto em parceiros mais definidos.

estudos da área de Medicina ele tem seus

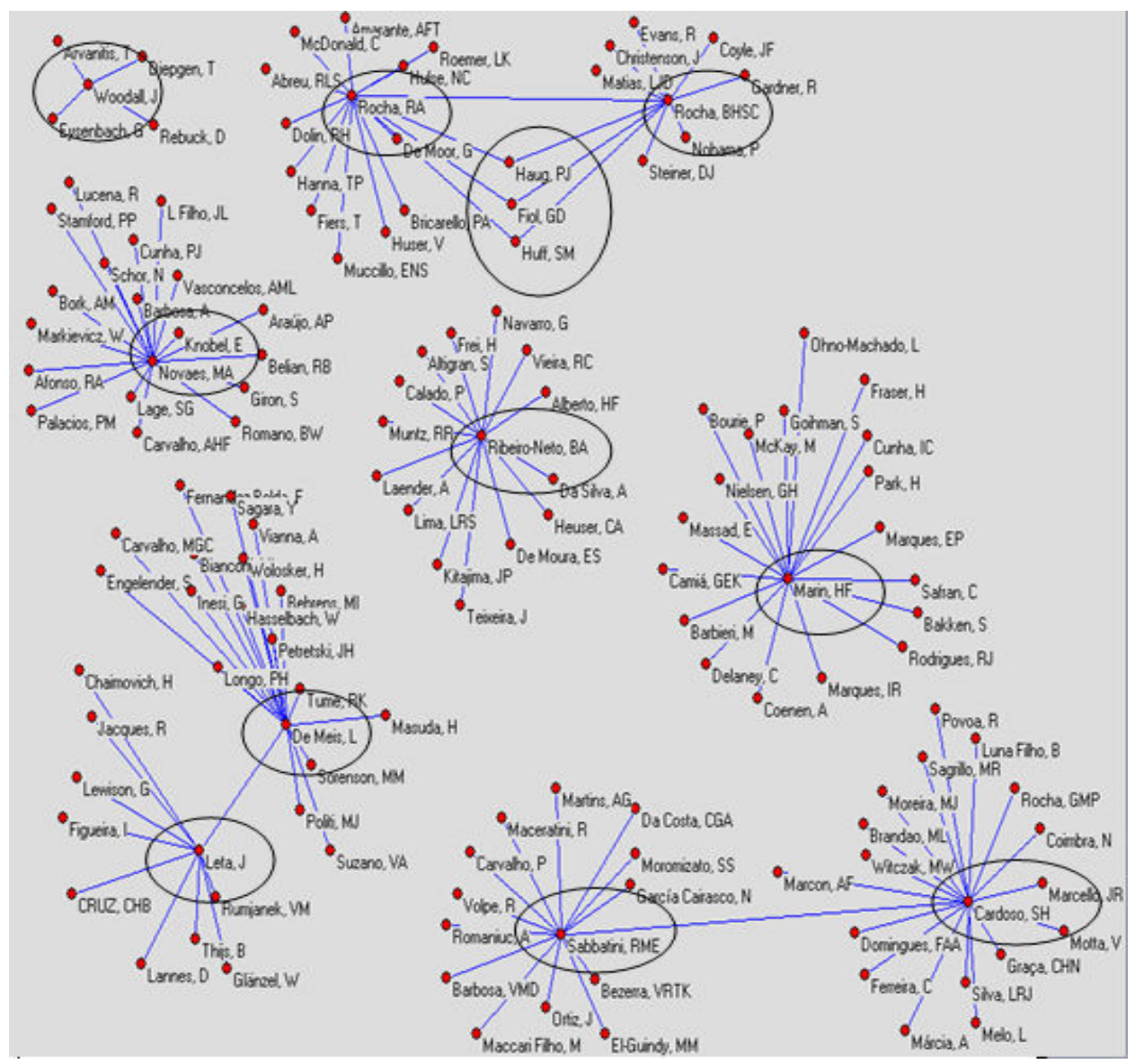

Figura 2 - Colaboração científica dos autores no Google Acadêmico.

Em comparação aos dois mapas podemos afirmar que o universo colaborativo do ISI foi mais interessante, por ter um grupo intermediário mais representativo e com uma importância na definição de identificar três autores que se colaboram mutuamente em função de seus "clusters". Porém o mapa de autores do Google Acadêmico deve ser imaginado com o mesmo "stress" do representado ao ISI com alguns acréscimos, mesmo porque a amostra para uma comparativa não foi sua exposição total, por questões de visualização.

Outro aspecto estudado foi o índice de citação recebido pelos autores, onde dividimos em duas etapas, contendo por um lado o total de citações recebidas por autoria, e por outro lado uma exposição dos documentos mais citados, sendo selecionados todos das bases de dados do ISI e os mais relevantes de cada autor para a análise no Google Acadêmico. 
A primeira análise está centrada pelo índice individual de autoria, no qual o ranking dentro de uma fonte foi diferente da outra.

Os principais resultados foram obtidos por Rocha, R.A.; Leta, J.; De Méis, L.; Ribeiro Neto, B.A. e Marin, H.F., que no item de citação todos estão entre os seis primeiros, em ambos recursos.

Este índice caracteriza que a maior centralidade das citações estão focadas nos estudos métricos (Bibliometria, Cienciometria e outras análises métricas), e nas áreas de ponta nacional (Medicina,
Biologia e Química), com uma influência de autores internacionais, como Glänzel, Rumjanek, Fiol, Huff, Witczak entre outros.

Se observarmos a Quadro 2, podemos identificar quatro tipos de autores citados, (i) os que tiveram um alto índice de citação (do $1^{\circ}$ ao $5^{\circ}$ no ranking do ISI); (ii) os que tiveram um moderado índice de citação em um dos recursos utilizados $\left(6^{\circ} \mathrm{e}\right.$ $7^{\circ}$ ); (iii) os que tiveram um índice nulo em ISI e um índice excelente no Google Acadêmico ( $8^{\circ}$ e $\left.10^{\circ}\right)$; e (iv) o autor que teve um índice baixo em ambos os recursos $\left(9^{\circ}\right)$, conforme representado no Quadro a seguir.

\begin{tabular}{|l|c|c|c|c|}
\hline \multirow{2}{*}{ Autores } & \multicolumn{2}{c|}{ ISI } & \multicolumn{2}{c|}{ Google School } \\
\cline { 2 - 5 } & Ranking & Citação & Ranking & Citados por \\
\hline Rocha, RA & 1 & 77 & 3 & 295 \\
\hline Leta, J & 2 & 53 & 6 & 170 \\
\hline De Meis, L & 3 & 38 & 1 & 2562 \\
\hline Ribeiro-Neto, BA & 4 & 28 & 2 & 420 \\
\hline Marin, HF & 5 & 11 & 5 & 201 \\
\hline Rocha, BHSC & 6 & 10 & 10 & 21 \\
\hline Woodall, J & 7 & 3 & 8 & 53 \\
\hline Sabbatini, RME & 8 & 0 & 7 & 168 \\
\hline Novaes, MD & 9 & 0 & 9 & 28 \\
\hline Cardoso, SH & 10 & 0 & 4 & 236 \\
\hline
\end{tabular}

Quadro 2 - Autores mais citados nas fontes de informação estudadas (ISI e Google Acadêmico).

Neste estudo não verificamos a questão da auto-citação, justamente porque não consideramos apropriado pelo fato de termos poucos registros recuperados no ISI e porque para uma análise mais relevante em este tipo de índice deveríamos contar com pelo menos 300 registros para uma adequada avaliação. Neste caso poderíamos também verificar o grau de centralidade dos autores em comparação com a auto-citação, verificando os passos de Cameo's (WHITE, 2001) da área, infelizmente ficará para outra pesquisa. 
Nossa seguinte análise relata o grau representado no Quadro 3.

de citação dos documentos, conforme

\begin{tabular}{|c|c|c|c|}
\hline Google Acadêmico & $\mathbf{Q t}$ & Bases de dados ISI & $\mathbf{Q t}$ \\
\hline $\begin{array}{l}\text { RIBEIRO NETO BA, 2002, ACM SIGMOD REC, } \\
\text { V31, P84 }\end{array}$ & 201 & $\begin{array}{l}\text { ROCHA RA, 1998, AM MED INFORM ASSOC, V5, } \\
\text { P276 }\end{array}$ & 48 \\
\hline $\begin{array}{l}\text { DEMEIS L, 1979, ANN REV BIOCHEM, V48, } \\
\text { P275 }\end{array}$ & 162 & $\begin{array}{l}\text { RIBEIRO NETO BA, 2002, ACM SIGMOD REC, } \\
\text { V31, P84 }\end{array}$ & 21 \\
\hline $\begin{array}{l}\text { DEMEIS L, 1981, } \\
\text { RETICULUM }\end{array}$ & 112 & $\begin{array}{l}\text { LETA J; DEMEIS L, 1996, SCIENTOMETRICS, } \\
\text { V35, P33 }\end{array}$ & 18 \\
\hline DEMEIS L, 1989, J BIOL CHEM, V264, P5929 & 93 & $\begin{array}{l}\text { ROCHA RA, 1998, AM MED INFORM ASSOC, V5, } \\
\text { P203 }\end{array}$ & 14 \\
\hline DEMEIS L, 1973, BIOCHEMISTRY, V12, P4581 & 77 & $\begin{array}{l}\text { ROCHA RA, 1995, AM MED INFORM ASSOC, V2, } \\
\text { P116 }\end{array}$ & 13 \\
\hline DEMEIS L, 1992, J BIOL CHEM, V267, P12606 & 73 & $\begin{array}{l}\text { LETA J; DEMEIS L, 1998, SCIENTOMETRICS, } \\
\text { V41, P313 }\end{array}$ & 10 \\
\hline $\begin{array}{l}\text { DEMEIS L, 1989, BIOCHIM BIOPHYS ACTA, } \\
\text { V973, P333 }\end{array}$ & 71 & $\begin{array}{l}\text { ROCHA BHSC, 2001, AM MED INFORM ASSOC, } \\
\text { V8, P117 }\end{array}$ & 8 \\
\hline $\begin{array}{l}\text { ROCHA, RA, 1998, AM MED INFORM ASSOC, } \\
\text { V5, P276 }\end{array}$ & 63 & $\begin{array}{l}\text { MARIN HF, 2001, AM MED INFORM ASSOC, V8, } \\
\text { P202 }\end{array}$ & 7 \\
\hline $\begin{array}{l}\text { RIBEIRO NETO BA, 1998, PROC THIRD ACM } \\
\text { CONF DIG LIBR, P182 }\end{array}$ & 62 & & \\
\hline $\begin{array}{l}\text { CARDOSO AH, 1994, NEUROSCI BIOBEHAV } \\
\text { REV, V18, P339 }\end{array}$ & 57 & & \\
\hline DEMEIS L;LETA J, 1996, PERFIL CIE BRASIL & 36 & & \\
\hline MARÍN HF, 1995, INFORMÁT EM ENFERMAG & 34 & & \\
\hline $\begin{array}{l}\text { RIBEIRO NETO BA, 1999, PROC STRING } \\
\text { PROC \& INF RET, P184 }\end{array}$ & 29 & & \\
\hline $\begin{array}{l}\text { RIBEIRO NETO BA, 2000, EXAMPLE-BASED } \\
\text { ENV WRAPPER GEN, V1921, P152 }\end{array}$ & 28 & & \\
\hline $\begin{array}{l}\text { CARDOSO AH, 1994, BEHAV BRAIN REV, } \\
\text { V63, P17 }\end{array}$ & 26 & & \\
\hline $\begin{array}{l}\text { LETA J; DEMEIS L, 1996, SCIENTOMETRICS, } \\
\text { V35, P33 }\end{array}$ & 24 & & \\
\hline $\begin{array}{l}\text { ROCHA, RA, 1994, COMP BIOMED RES, V27, } \\
\text { P472 }\end{array}$ & 24 & & \\
\hline $\begin{array}{l}\text { CARDOSO AH, 1993, BEHAV BRAIN REV, } \\
\text { V58, P49 }\end{array}$ & 23 & & \\
\hline $\begin{array}{l}\text { CARDOSO AH, 1992, PHYSIOL BEHAV, V51, } \\
\text { P557 }\end{array}$ & 20 & & \\
\hline $\begin{array}{l}\text { ROCHA RA, 1998, AM MED INFORM ASSOC, } \\
\text { V5, P203 }\end{array}$ & 18 & & \\
\hline $\begin{array}{l}\text { ROCHA BHSC, 2001, AM MED INFORM } \\
\text { ASSOC, V8, P117 }\end{array}$ & 16 & & \\
\hline $\begin{array}{l}\text { MARÍN HF, 2001, AM MED INFORM ASSOC, } \\
\text { V8, P202 }\end{array}$ & 16 & & \\
\hline $\begin{array}{l}\text { ROCHA RA, 1995, AM MED INFORM ASSOC, } \\
\text { V2, P116 }\end{array}$ & 15 & & \\
\hline $\begin{array}{l}\text { LETA J; DEMEIS L, 1998, SCIENTOMETRICS, } \\
\text { V41, P313 }\end{array}$ & 14 & & \\
\hline LETA J, 2002, SCIENTOMETRICS, V53, P325 & 13 & & \\
\hline LETA J, 2001, SCIENTOMETRICS, V50, P241 & 10 & & \\
\hline $\begin{array}{l}\text { SABBATINI RME, 1994, REV INFORMEDICA, } \\
\text { V1, P5 }\end{array}$ & 9 & & \\
\hline $\begin{array}{l}\text { SABBATINI RME, 1999, REV MED REP, V2, } \\
\text { P20 }\end{array}$ & 9 & & \\
\hline
\end{tabular}

Quadro 3 - Documentos mais citados. 
Este processo esta muito relacionado com o índice de autoria (Quadro 2), em especial porque os autores são referências deste trabalho, principalmente no caso de De Meis e Ribeiro Neto, que tiveram em um único documento, de ambos os recursos, um total mais elevado do que os autores Rocha BHSC, Woodall, Sabbatini, Novaes e Cardoso.

Nesta análise buscamos representar os trabalhos mais citados pelos autores em questão, no qual alguns tiveram um índice baixo, com 9 ou 10 citações. Entretanto no caso de De Meis, Rocha RA e Ribeiro Neto tivemos um índice de vários trabalhos entre 20 a 30 citações e não mencionamos na Quadro anterior para não ficar uma influência científica somente em três ou quatro autores.

Todos os registros citados em ISI foram também representados no Google Acadêmico e com um índice mais elevado. Isto ocorre pelo fato de que muitos dos autores brasileiros citam em revistas que não estão indexadas no ISI, o mesmo não ocorreu nas bases de dados do Institute for Scientific Information, ao qual o índice de citação faz parte de documentos também indexados no ISI, uma forma de controlar melhor este grau científico por parte dos especialistas da Web of Knowledge.

Como totalidade de documentos, podemos dizer que as revistas Scientometrics, Journal of the American
Medical Intormatıcs Associatıon e Journal Biological Chemical foram os principais meios de citação, em parte pela influência da área de Medicina (autores da Unicamp), da Biologia (IHC, UFPr e UFMG) e das Biomédicas representadas em forma de estudos métricos (UFRJ); e em outra aspectos porque estas revistas mencionadas são de grande qualidade e de uma divulgação importante ao meio acadêmico.

Finalmente, o índice dentro do Google Acadêmico teve uma constante de todos os autores, onde o mesmo não aconteceu em ISI, com autores sem nenhuma citação. O processo do Google Acadêmico facilita e reafirma a constância científica dos autores, em especial da área de Ciência da Informação, mostrando que além dos meios tradicionais (as bases de dados especializadas) podemos também contar com um índice multidisciplinar para avaliar a ciência nacional e seu índice de citação, contando com várias tipologias além do tradicional artigo de revistas.

\section{CONCLUSÃO}

Este estudo introduz uma nova forma de analisar as informações científicas, abordando o Google Acadêmico como um recurso viável e com um índice elevado da produtividade e da citação dos autores da área de Ciência da Informação. Os resultados indicam que este recurso é 
comparativo com o empregado pelas bases de dados do ISI, onde o ranking dos cinco primeiros é muito equivalente e por este motivo sua relevância é necessária para a área no Brasil em novos estudos.

Algumas das diferenças em relação aos dois recursos são devido ao fato de que a estratégia de recuperação e de indexação das bases de dados do ISI não são de todo confiável, principalmente em nomes compostos e ápodos nos sobrenomes. O Google Acadêmico não possui uma política desobstruída, mas ao contrário do ISI posiciona a recuperação de registros de livros, comunicações e documentos postados na web. Outro aspecto importante é o índice de citação, conduzindo o pesquisador aos documentos que se encontram as citações ou a documentos similares.

Como recomendação, sugerimos que uma investigação dentro do Google deve ser de âmbito comparativo com outras bases de dados para ver a sua influência e em que medida existe uma similaridade de dados e registros. $E$ por outro lado, podemse comparar seus documentos indexados com outros motores de busca de quarta geração, como Scirus e Academic Microsoft Live.

\section{REFERÊNCIAS}

BAR-ILAN, Judit; LEVENE, Mark; LIN, Ayelet. Some measures for comparing citation databases. Journal of Informetrics, Amsterdam, v. 1, p. 26-34, 2007.

BATAGELJ, Vladimir; MRVAR, Andrej. Pajek analysis and visualization of large networks. Preprint Series, Ljubljana, v. 41, n. 871, p. 2-26, 2003.

CAÑEDO ANDALIA, Rubén. Estudio de solapamiento en la selección de las publicaciones seriadas y las bases de datos. ACIMED, La Habana, v.7, n.3, p.164-170, 1999a.

CAÑEDO ANDALIA, Rubén. Los análisis de citas en la evaluación de los trabajos científicos y las publicaciones seriadas. ACIMED, La Habana, v. 7, n. 1, p.30-39, 1999b.

GARFIELD, Eugene. Citation Indexes for Science: A New Dimension in Documentation through Association of Ideas. Science, Washington, v.122, n.3159, p.108-111, 1955. Disponível em:

<http://www.garfield.library.upenn.edu/essa ys/v6p468y1983.pdf>. Acesso em: 31 jul. 2007.

GOOGLE. Google School support for scholarly publishers. 2005. Disponível em: $<$ http://scholar.google.com/scholar/publishe rs.html>. Acesso em: 31 jul. 2007.

GOTO, Nelly; COTLER, Emily. Rediseño y desarrollo de sitios web. A Coruña: Anaya, 2005.

GUTIERREZ GALLARDO, Juan Diego; LÓPEZ GUISADO, Ángel. Google. A

Coruña: Anaya, 2005.

JACSO, Peter. As we may search: comparison of major features of Web of Science, Scopus and Google School citation-based and citation-enhanced databases. Current Science, Bangalore, v.89, n.9. p.1537-1547, 2005. 
LELOUP, Catherine. Motores de búsqueda e indización. Barcelona: Gestión 2000, 1998.

MARCHIONINI, Gary. Information seeking in electronic environments. Cambridge: University Press, 1995.

MOLINA, José Luis; MUÑOZ, Juan M.; DOMENECH, Miquel. (2001). Redes de publicaciones científicas: un análisis de la estructura de coautorías. In: XXI

SUNBELT, Budapest, 2001. Disponível em: $<$ http://seneca.uab.es/antropologia/jlm/publi c archivos/copub.pdf>. Acesso em: 31 jul. 2007.

NORUZI, Alireza. Google Scholar: the new generation of citation indexes. LIBRI, Copenhagen, v.55, n.4, p.170-180, 2005.

PRICE, Gary. Google Scholar goes international. In: SerachEngineWatch Blog. Disponível em:

$<$ http://blog.searchenginewatch.com/blog/0 60111-145555>. Acesso em: 18 jun. 2007.

SAXTON, Matthew L. ProCite 5.0. The Library Quarterly, Chicago, v. 71, n. 2, p. 288-290, 2001.

WHITE, Howard D. Author-centered bibliometrics through CAMEOs: characterizations automatically made and edited online. Scientometrics, Budapest, v. 51, n. 3, p. 607-637, 2001.

\section{Title}

Scientific index of the Information Science: a comparative study between the authors with visibility in ISI and Google Scholar.

\section{Abstract}

This work aimed to analyze the Information Science main authors' productivity at ISI and Google Scholar, using a methodology of information recovery by countries, followed by sub-categories (ISI) recovery and by authoring (Google School). For data refining it was used the ProCite software for ISI and for the web environment it was chosen the FlasGet. Regarding the main results it is highlighted that the UFRJ and Unicamp have an excellent scientific production; the UFRJ authors' represented a huge amount of citation index and it was observed that the authors analyzed have a great scientific collaboration represented by Social Web map.

\section{Keywords}

Scientific Visibility; ISI; Google Scholar; Citation Analysis; Social Network.

\section{Titulo}

Contenido científico de la ciencia de la información: comparativo de los autores más visibles en el ISI y en el Google Académico.

\section{Resumen}

El objetivo del estudio fué analisar la productividad de los principales autores del área de ciencias de la información en el ISI y en el Google Académico, utilizando un método de recuperación de información por países, seguido por un filtro de subcategorías (ISI) y autores (Académico). Para el refinamiento de los datos utilizamos el ProCite, para el ISI y el FlashGet, para el ambiente web (google). Se destacan como resultados más importantes la excelente productividad de los autores de la UFRJ y la Unicamp, los altos indicadores de las citas de los autores de la UFRJ y el alto grado de colaboración científica de los mismos, representados como mapas de las redes sociales.

\section{Palabras clave}

Visibilidad la ciência; ISI; Google Académico; Análisis de citas; Red Social.

Recebido em: 13/12/2007

Aceito em: 19/06/2008 